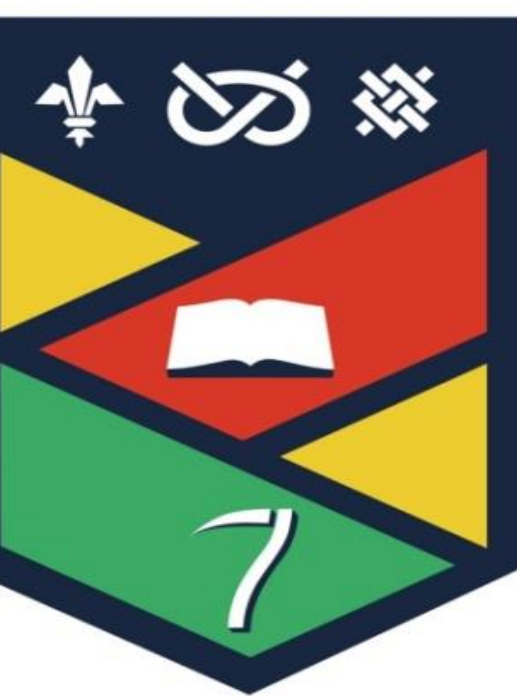

Keele University
Challenges in anticoagulation therapy following ischemic stroke in patients with atrial fibrillation

Keskes $\mathbf{S}^{1}$, Sanyal $\mathbf{R}^{2}$, Muddegowda $\mathbf{G}^{2}$, Natarajan $\mathbf{I}^{2}$, Chembala $\mathrm{C}^{2}$, Roffe $\mathbf{C}^{2}$ 1 Medical Student Keele University, UK, 1 University Hospital of North Midlands NHS Trust, Stroke Medicine, Stoke-on-Trent
And Stroke Research in Stoke, Institute for Applied Clinical Studies, Keele University, UK

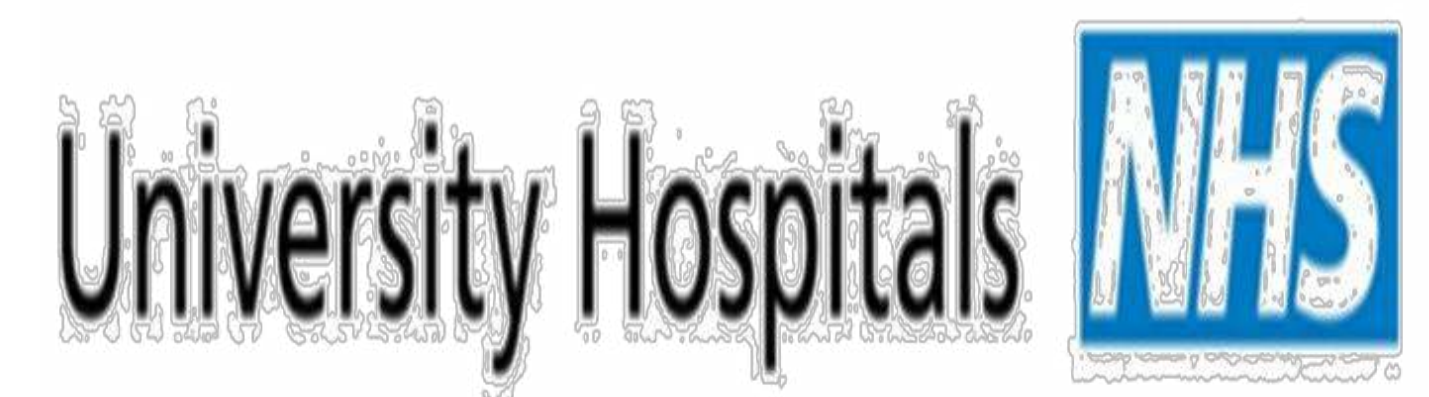

\section{of North Midands}

NHS Trust

\title{
INTRODUCTION
}

Atrial fibrillation (AF), is the most significant risk factor for ischaemic stroke, with an increased risk of almost sevenfold, with more than $20 \%$ of strokes in England and Wales resulting secondary to $\mathrm{AF}^{1}$. Patients are also at high risk of recurrent stroke $e^{2}$,poorer neurological Patients are also at high risk of recurrent stroke', poorer
outcome, increased mortality and longer hospital stays.

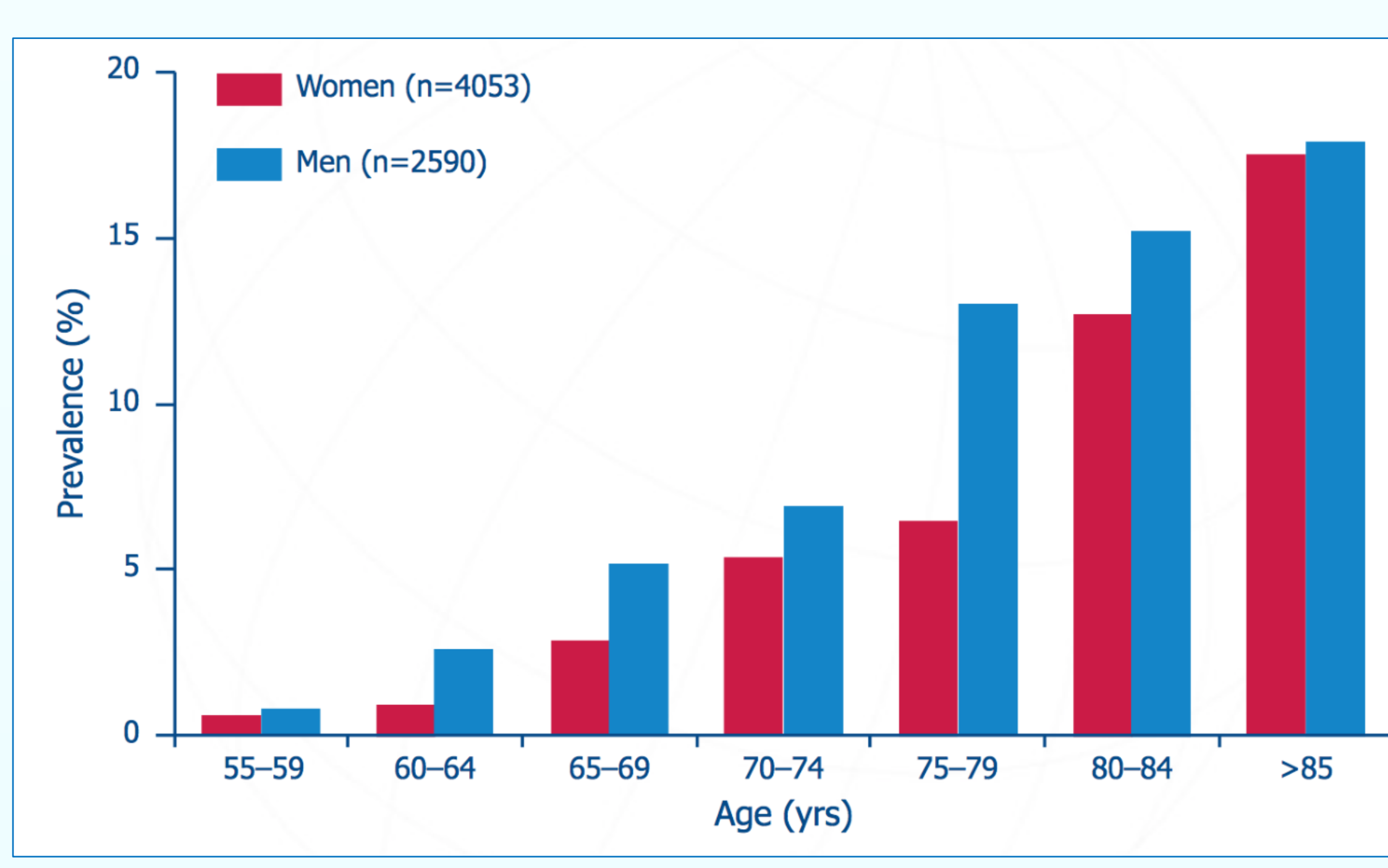

Oral anticoagulation (OA) has been proven to be an effective risk reducing treatment, however its optimal timing is under constant review, as it appears that the the most favorable outcome is largely patient specific. It has been shown that patients with early recanalization have better outcomes at 3 months and lower mortality however, in patients presenting with $A F$, the early initiation of $O A$ is delayed due to an increased risk of haemorrhagic transformation, particularly intracerebral haemorrhage (ICH)

Currently, the most important and commonly used scheme to stratify the risk of stroke in AF patients comprises the CHA2DS2-VASc score a development of the earlier CHA2DS2 criteria yet now being more inclusive. This scheme incorporates additional risk factors for stroke, classifying patients into low to high risk categories for which subsequent management is directed accordingly. This can be validated alongside the HAS-BLED scoring system which is one of the multiple tools used to evaluate risk of bleeding in AF patients. Together, these can be used synonymously to identify correct management and importantly, address any reversible risk factors identified which can aid recovery.
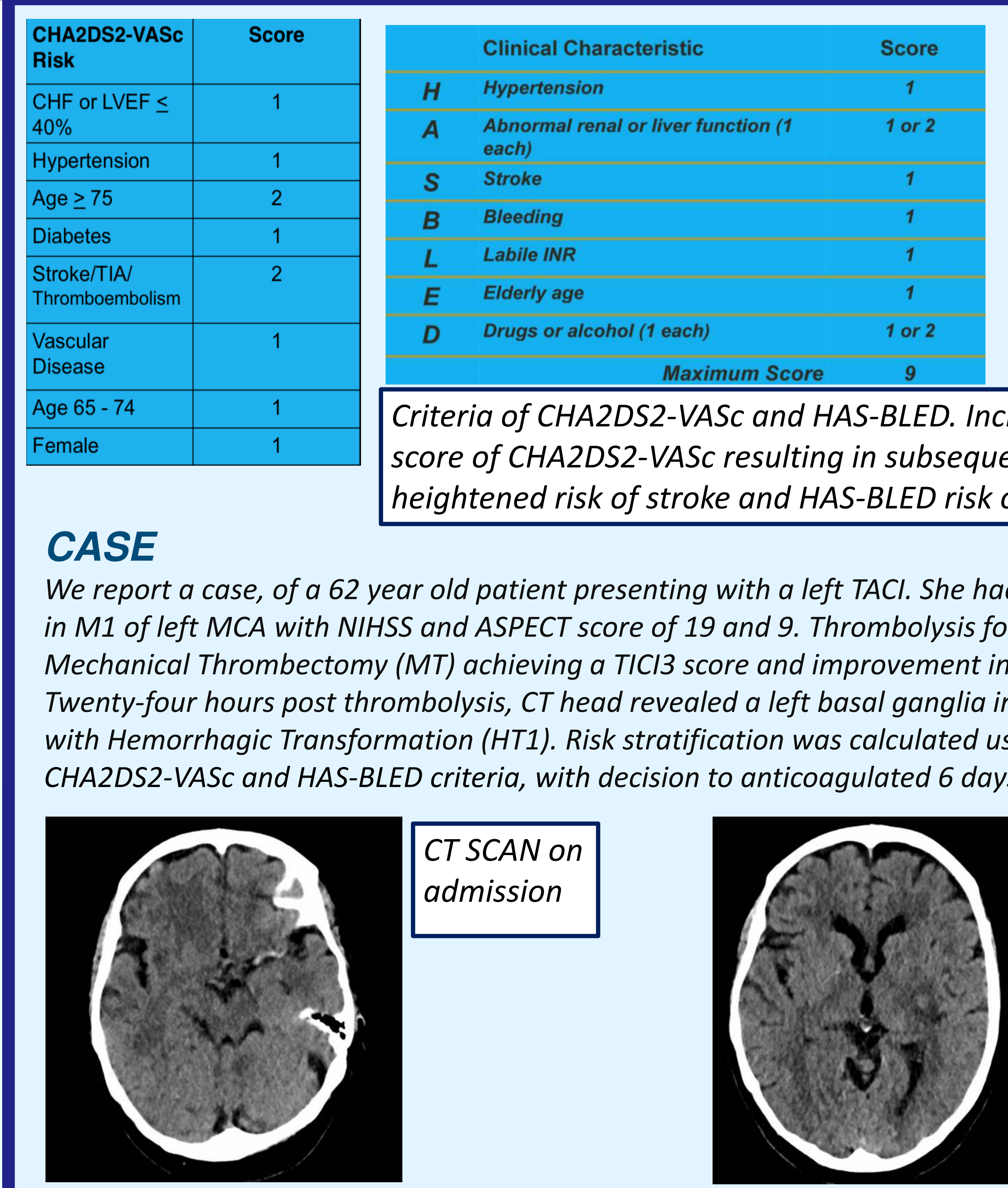

Five days later, the patient suffered a right TACl, with an NIHSS of 17 and thrombus in M1 segment of the right MCA. As thrombolysis was contraindicated, MT was repeated and TICI3 flow achieved. NIHSS reduced to 5 with repeat CT confirming a $1.5 \mathrm{~cm}$ infarct within the right lentiform nucleus. With high risk of further embolic episodes, split dose low molecular weight heparin was initiated to reduce the risk of further embolic stroke.

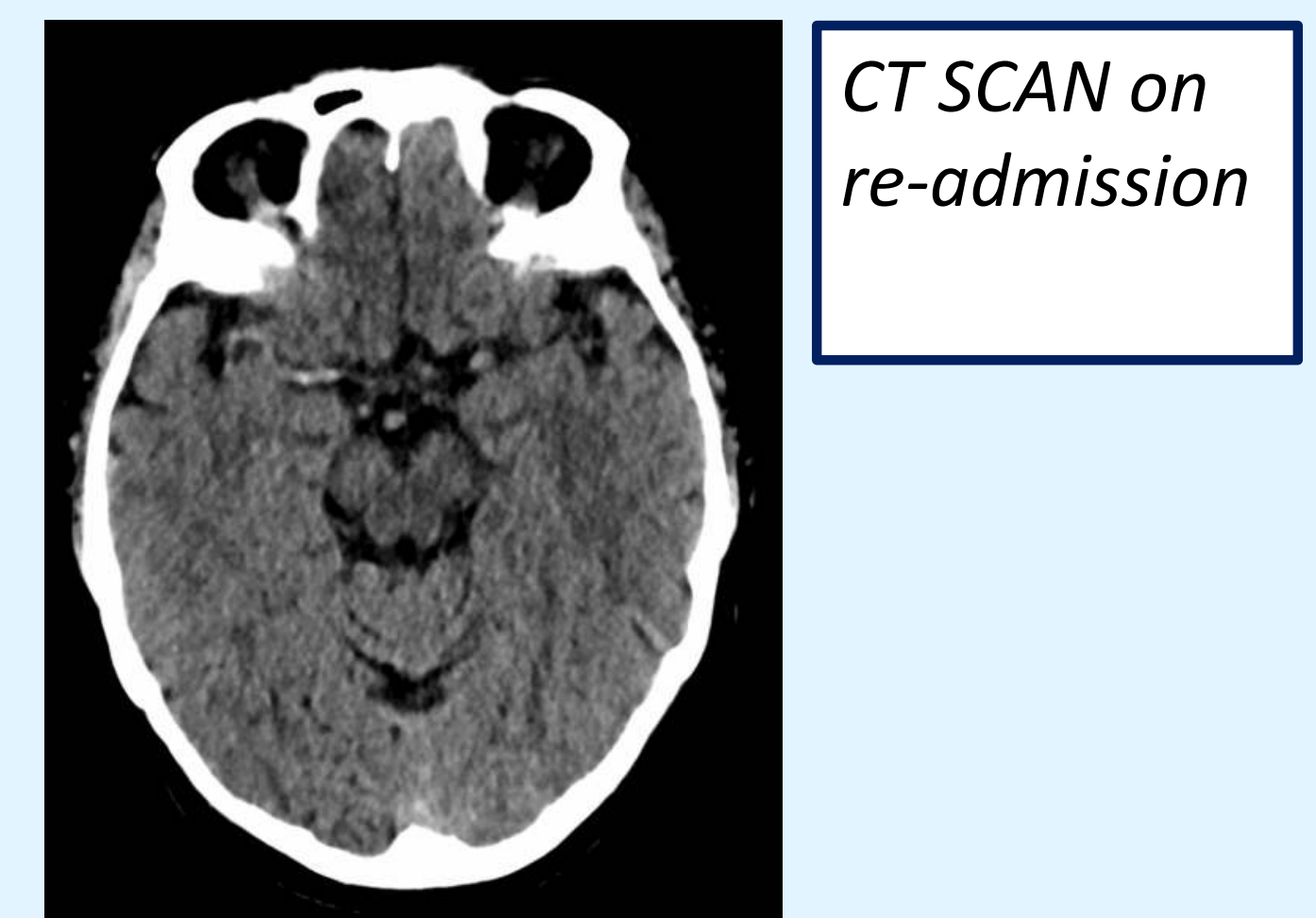

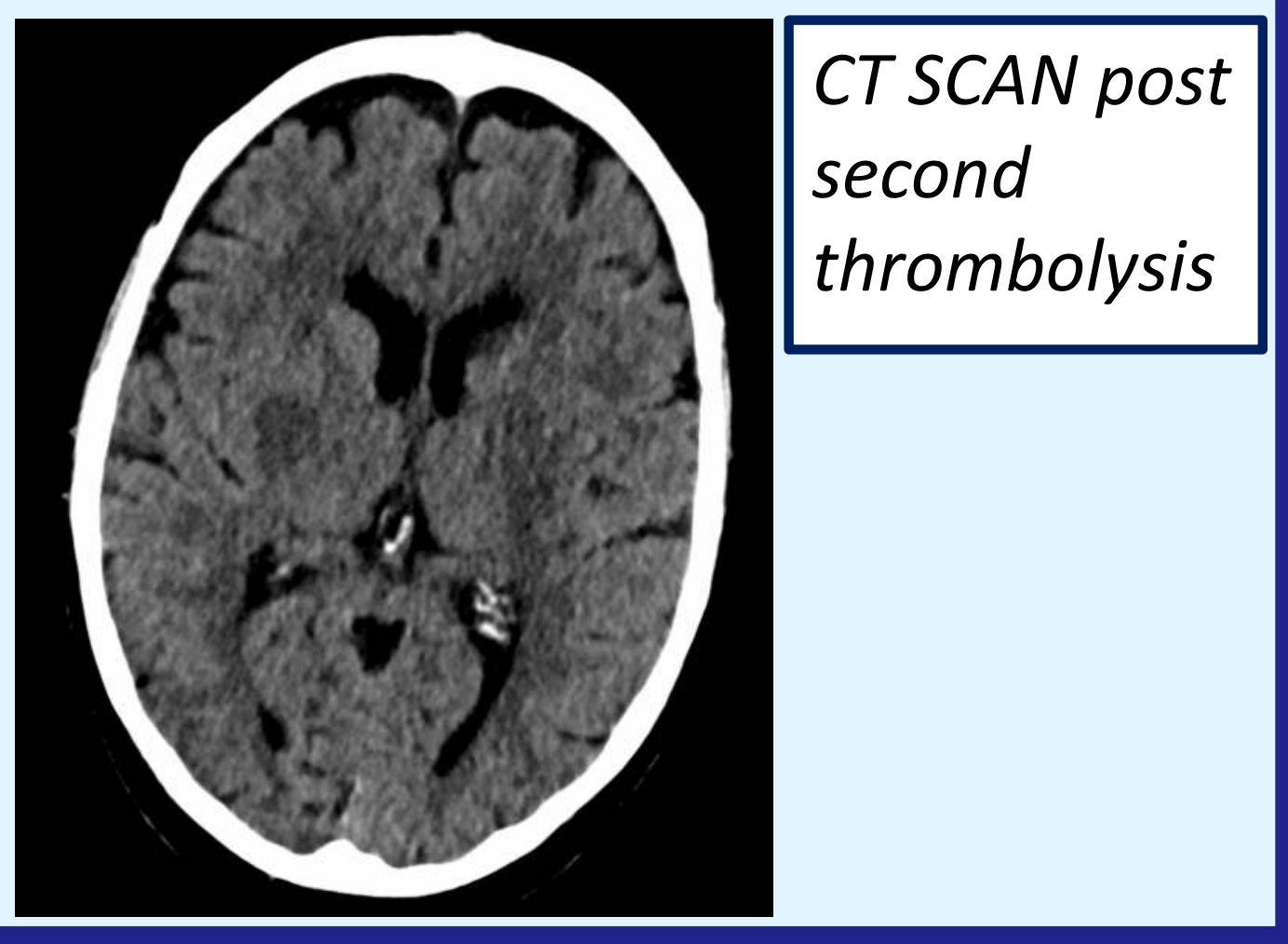

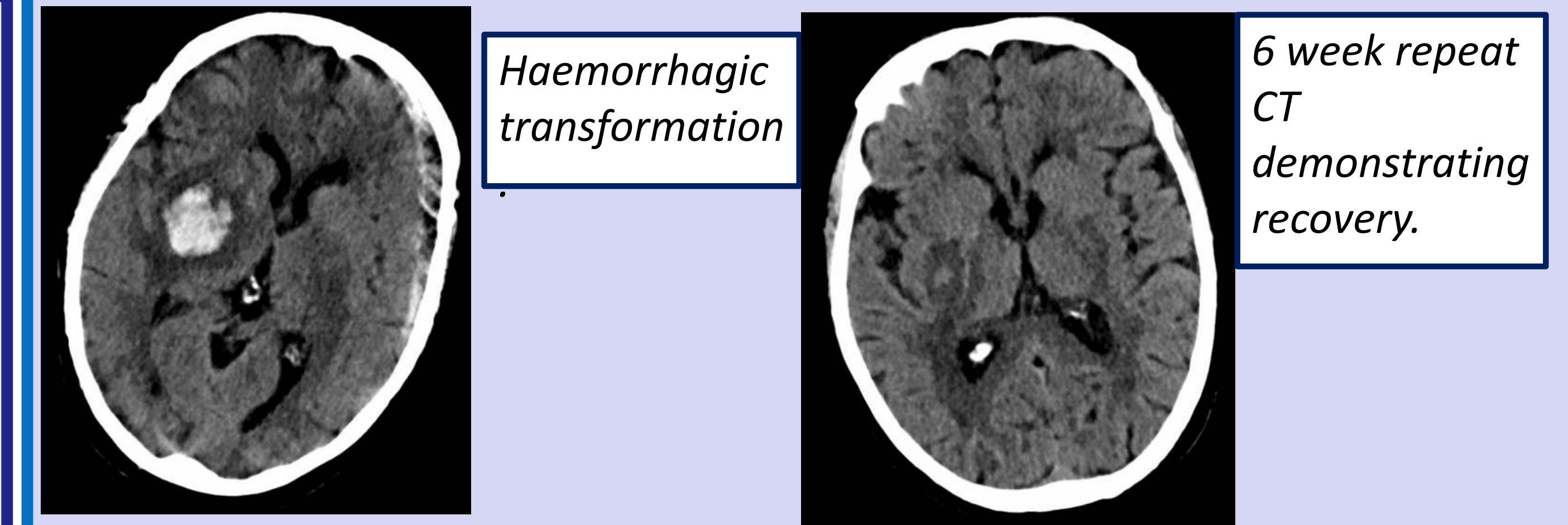

Three days later, NIHSS deteriorated to 19 and CT head confirmed Parenchymal Hemorrhage (PH1). Anticoagulation was stopped and six weeks rehabilitation followed, reducing NIHSS to 4 and MRS to 2. With resolution of the Haemorrhage, oral anticoagulation with Apixaban was resumed. Three months follow up showed NIHSS and MRS of 1.

\section{DISUSSION AND CONCLUSION}

The timing of oral anticoagulation following stroke is always difficult, but this case highlights the unpredictable nature of stroke especially in patients with AF. Primary prevention of stroke in these patients is crucial as this can aid in the decline of mortality and morbidity from stroke which can be extremely complexed, as this case highlights. Moreover, thrombectomy is able to be performed in both instances with positive outcome. Application of the guidelines to take decisions in

thromboprophylaxis fundamental for clinical benefit and net reduction in mortality. But, although there are risk stratification systems in place, there is a need to assess each patient on an individualistic basis prior to forming a decision. The guidelines must be used alongside clinician experience and the evaluation of the patient's condition including pre-stroke morbidity. This allows evaluation of patients on a case specific nature, aiming to reduce risk of recurrence and improve overall quality of life.

REFERENCES

1. López-López José A, Sterne Jonathan A C, Thom Howard H Z, Higgins Julian P T, Hingorani Aroon $\mathrm{D}$, Okoli George $\mathrm{N}$ et al. Oral anticoagulants for prevention of stroke in atrial fibrillation. systematic review, network meta-analysis, and cost effectiveness analysis BMJ 2017; 359 :j5058

Hart RG Secondary Prevention of Stroke in Patients with Atrial Fibrillation Practical Neurology 2003;3:260-267.

Heeringa, J., van der Kuip, D., Hofman, A., Kors, J., van Herpen, G., Stricker, B., Stijnen, Lip, G. and Witteman, J. (2018). Prevalence, incidence and lifetime risk of atrial fibrillation: the Rotterdam study.

Von Kummer R, Holle R, Rosin L, Forsting M, Hacke W. Does arterial recanalisation improve outcome in carotid territory stroke? Stroke 1995; 26:581-587.

. Åsberg S, Hijazi Z, Norrving B, Terént A, Öhagen P, Oldgren J. Timing of oral anticoagulant therapy in acute ischemic stroke with atrial fibrillation: study protocol for a registry-based randomised controlled trial. Trials. 2017;18:581. 